\title{
Modified 3-Step Broadband Light Treatment for Inflammatory Acne Vulgaris and Post-Acne Erythema in Asian Patients
}

Yuko Ito'

Sung Bin $\mathrm{Cho}^{2,3}$

${ }^{1} Y$ 's Skin Care Clinic, Miyagi Prefecture, Japan ${ }^{2}$ Department of Dermatology and Cutaneous Biology Research Center, International St. Mary's Hospital, Catholic Kwandong University College of Medicine, Incheon, Korea

${ }^{3}$ Kangskin Sillim Dermatology Clinic, Seoul, Korea
Received December 15, 2017

Revised December 18, 2017

Accepted December 18, 2017

\section{Correspondence}

Sung Bin Cho

Department of Dermatology and Cutaneous

Biology Research Center, International St. Mary's Hospital, Catholic Kwandong University College of Medicine, 25 Simgok-ro, Seo-gu, Incheon 22711, Korea

Tel.: +82-32-290-3141

Fax: $+82-32-290-3142$

E-mail: drsbchođggmail.com

(c) Korean Society for Laser Medicine and Surgery

(c) This is an open access article distributed under the terms of the Creative Commons Attribution NonCommercial License (http://creativecommons.org/ licenses/by-nc/4.0) which permits unrestricted noncommercial use, distribution, and reproduction in any medium, provided the original work is properly cited.
Light and laser sources have been used as additive or alternative therapeutic modalities for the treatment of active acne vulgaris with or without the use of topical photosensitizers. Early intervention on inflammatory lesions has been deemed to reduce the risks of post-acne erythema and scarring. A novel 3-step protocol was introduced to treat inflammatory acne lesions and scarring by delivering broadband light (BBL) at three different treatment settings. This paper describes the successful treatment of two Japanese patients with active acne vulgaris and post-acne erythema using a modified 3-step BBL treatment protocol. The modified approach comprised of treatment with a cut-off filter of $420 \mathrm{~nm}$ and a pulse duration of $150 \mathrm{msec}$ (step 1), a cut-off filter of $590 \mathrm{~nm}$ and a pulse duration of $200 \mathrm{msec}$ (step 2), and a cut-off filter of $590 \mathrm{~nm}$ and a pulse duration of $12 \mathrm{sec}$ (step 3). One month after the last treatment, both patients exhibited marked reductions in inflammatory acne lesions and post-acne erythema. The overall skin tone and texture were also improved after the 3-step BBL treatment without major side effects.

\section{Key words}

Acne vulgaris; Broadband light; 3-step protocol; Cut-off filter; Acne scar 


\section{INTRODUCTION}

Light and laser sources have been used as additive or alternative therapeutic modalities for treating acne vulgaris with or without the use of topical photosensitizers. ${ }^{1-4}$ The mechanism of action thereof is theoretically based on the selective photothermolysis of factors contributing to the pathogenesis of acne vulgaris. ${ }^{2-4}$ Thereby, microorganisms, pilosebaceous units, and surrounding dermal inflammatory and vascular components can be modified or destroyed..$^{2-4}$ Early intervention using light and laser sources for treating inflammatory acne lesions has also been deemed to reduce the risks of post-acne erythema and scarring. ${ }^{3,4}$

In a previous report, a novel 3-step protocol designed to deliver broadband light (BBL) in three different treatment settings was introduced for treating inflammatory acne.' In step 1, the BBL treatment settings comprise a cut-off filter of $420 \mathrm{~nm}$, a fluence of $4-6 \mathrm{~J} / \mathrm{cm}^{2}$, a pulse duration of 240-300 msec, and 3 passes over active acne lesions to induce photobiologic reaction by targeting porphyrins in Propionibacterium acnes.' In step 2, BBL is delivered at the treatment settings of a cut-off filter of 560 or 590 $\mathrm{nm}$, a fluence of $15 \mathrm{~J} / \mathrm{cm}^{2}$, a pulse duration of $15 \mathrm{msec}$, and 2-3 passes over active acne lesions and scars to reduce inflammatory reactions and to induce new collagen production. ${ }^{1}$ Finally, in step 3 , the $\mathrm{BBL}$ treatment settings consist of a cut-off filter of $590-1200 \mathrm{~nm}$, an intensity of 8-15 W/ $\mathrm{cm}^{2}$, a pulse duration of $12 \mathrm{sec}$, and a constant motion technique over active acne lesions and potential breakout areas. ${ }^{1}$

In the present study, we describe two Japanese patients with active acne vulgaris and post-acne erythema who were successfully treated with a modified 3-step BBL treatment protocol. The modified 3-step protocol comprised treatment with a cut-off filter of $420 \mathrm{~nm}$ and a pulse duration of $150 \mathrm{msec}$ (step 1), a cut-off filter of 590 $\mathrm{nm}$ and a pulse duration of $200 \mathrm{msec}$ (step 2), and a cutoff filter of $590 \mathrm{~nm}$ and a pulse duration of $12 \mathrm{sec}$ (step 3).

\section{CASE REPORT}

Two Japanese patients (14-year-old and 22-year-old females) visited our clinic presenting with erythematous pustular lesions on the forehead, perioral area, and cheeks. The patients had been treated with oral and topical medications at other clinics, although the clinical outcomes thereof were unsatisfactory. Neither patient had undergone pertinent light- or laser-based treatments, such as pulsed dye laser, intense pulsed light, or other long-pulsed lasers, for inflammatory acne vulgaris, postacne erythema, and postinflammatory hyperpigmentation within the last three months. The patients were clinically diagnosed with inflammatory acne vulgaris (Fig. 1A and 2A), and were prescribed topical adapalene gel to reduce inflammatory acne lesions for 1 to 2 months. However, despite partial improvement thereof, the active inflammatory lesions and post-acne erythema persisted.

After obtaining written informed consent, the patients were treated with two (14-year-old patient) and four ses-
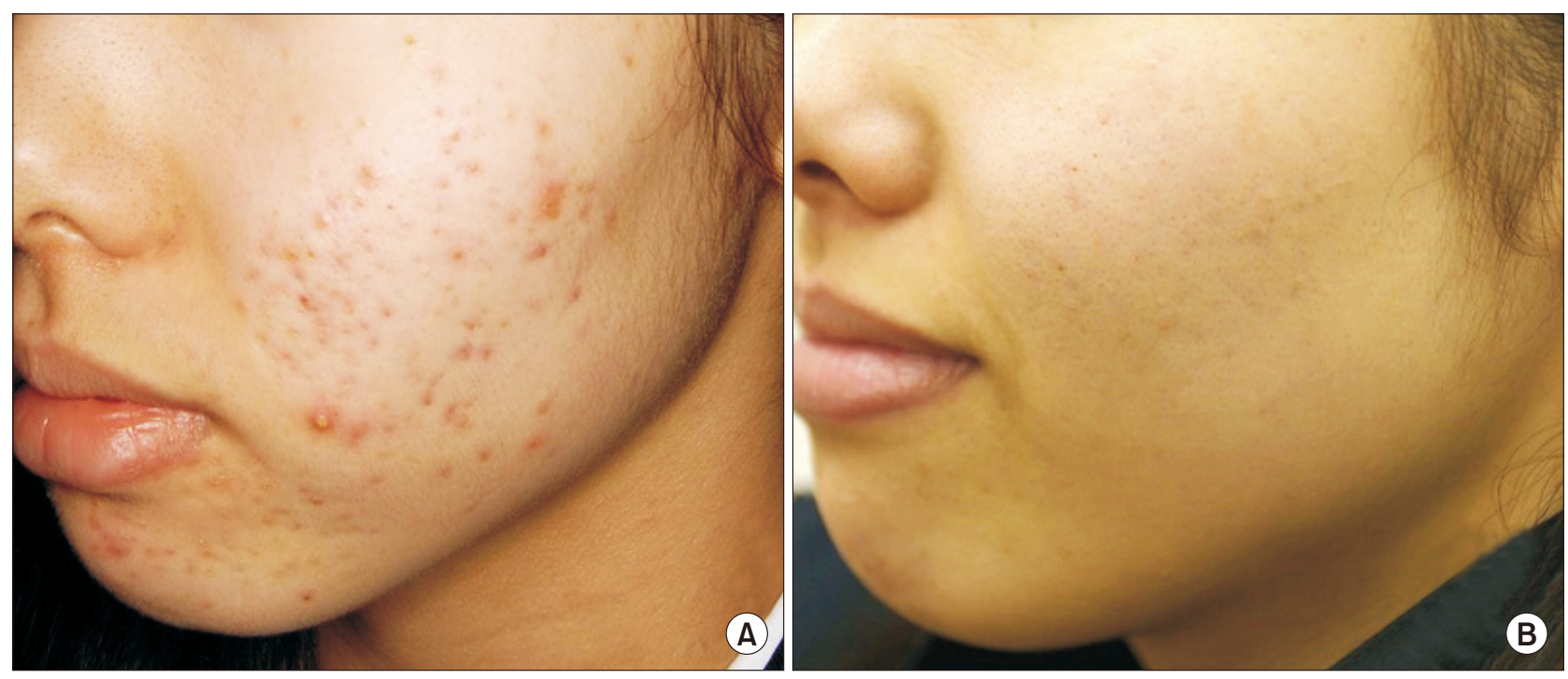

Fig. 1. Photographs of a 14-year-old female patient. The patient was treated with two sessions of modified 3-step broadband light (BBL) treatment for inflammatory acne vulgaris at 4-week intervals. (A) Baseline and (B) one month after the second session of 3-step BBL treatment. 

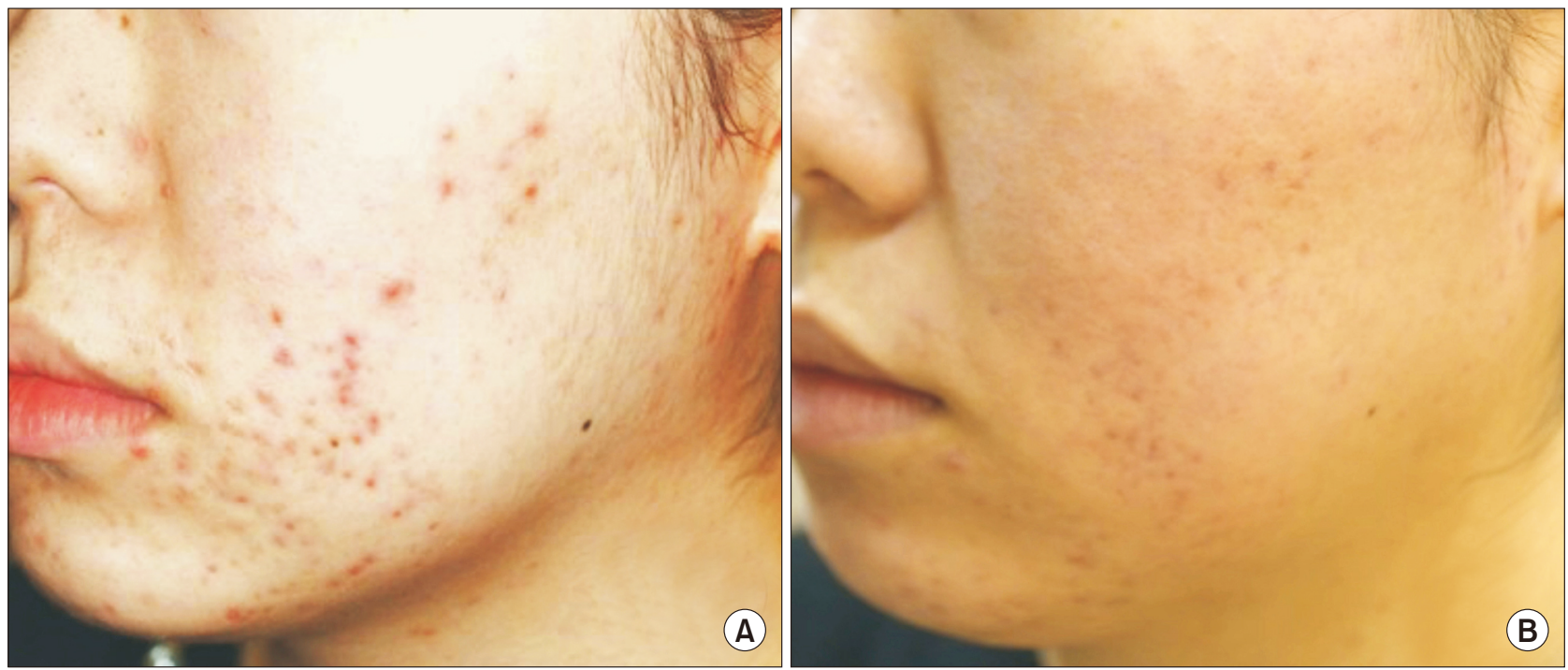

Fig. 2. Photographs of a 22-year-old female patient. The patient was treated with four sessions of modified 3-step BBL treatment for inflammatory acne vulgaris at 4-week intervals. (A) Baseline and (B) one month after the fourth session of 3-step BBL treatment.

sions (22-year-old patient) of 3-step BBL treatment for inflammatory acne vulgaris and post-acne erythema at one-month intervals. The face of each patient was gently cleansed with a mild soap, and chilled ultrasonic gel was applied on the entire face without pretreatment with topical anesthetic cream. Then, the 3-step BBL treatment was performed as follows: In step 1, a single pass of BBL treatment (BBL; Sciton, Palo Alto, CA, USA) with a cut-off filter of $420 \mathrm{~nm}$, a fluence of $5 \mathrm{~J} / \mathrm{cm}^{2}$, a pulse duration of $150 \mathrm{msec}$, and a cooling crystal temperature of $15^{\circ} \mathrm{C}$ was delivered on the entire face after applying chilled ultrasonic gel. In step 2, a single additional pass of BBL treatment with a cut-off filter of $590 \mathrm{~nm}$, a fluence of $15 \mathrm{~J} / \mathrm{cm}^{2}$, a pulse duration of $200 \mathrm{msec}$, and a cooling crystal temperature of $15^{\circ} \mathrm{C}$ was applied on the entire face. In step 3 , BBL treatment was performed on the face with a 590ST cut-off filter (Sciton), an intensity of $10 \mathrm{~W} / \mathrm{cm}^{2}$, a pulse duration of $12 \mathrm{sec}$, and a cooling crystal temperature of $30^{\circ} \mathrm{C}$ via a constant motion technique. The estimated total accumulation energy of the step 3 treatment was approximately $25,000 \mathrm{~J} /$ session. The treated areas were cooled with icepacks. No prophylactic systemic or topical corticosteroids, antivirals, or antibiotics were prescribed. The patients were recommended to apply sunscreen and topical adapalene gel thereafter.

One month after the final treatment, both patients exhibited marked reductions in inflammatory acne lesions and post-acne erythema (Fig. 1B and 2B). Furthermore, overall skin tone and texture were improved. The patients were very satisfied with the results, and reported that sebum secretion had noticeably decreased. Pain during the treatment was tolerable without the use of topical anesthetic cream, and most of the post-treatment erythema spontaneously disappeared within two hours. Furthermore, no remarkable major side effects, including crusts, oozing, petechiae or bruising, bleeding, burn, worsening of inflammatory acne lesions, prolonged edema or erythema, post-therapy dyschromia, and scarring, were encountered.

\section{DISCUSSION}

Laser- and light-based therapies exerts beneficial effects on active acne lesions by inducing photobiologic reactions on one or more of the key pathogenic factors thereof, including complex inflammatory reaction, follicular hyperkeratinization, $P$. acnes colonization, and excessive sebum production. ${ }^{3,5}$ However, although various pulsed dye, diode, potassium titanyl phosphate, and carbon dioxide laser devices, radiofrequency systems, and BBL systems have been used, further randomized controlled studies are needed for optimizing laser- and light treatment settings for acne vulgaris. ${ }^{3,5}$ In the present study, we discovered that two Japanese patients with active acne vulgaris and post-acne erythema experienced satisfactory improvement therein after treatment with a modified 3-step BBL protocol without the use of topical photosensitizers.

The modified 3-step approach applied in this study comprised treatment with a cut-off filter of $420 \mathrm{~nm}$ and a 
pulse duration of 150 msec (step 1), a cut-off filter of 590 $\mathrm{nm}$ and a pulse duration of $200 \mathrm{msec}$ (step 2), and a cutoff filter of 590ST and a pulse duration of $12 \mathrm{sec}$ (step 3). The use of blue light at a wavelength of 407-420 nm can eradicate $P$. acnes, an organism that has been found to induce complex inflammatory reactions and to contribute to the pathogenesis of acne vulgaris. ${ }^{6}$ Furthermore, BBL treatment with the cut-off wavelength of $420 \mathrm{~nm}$ has been shown clinically to reduce the number of inflammatory acne lesions and to elicit anti-inflammatory effects by downregulating tumor necrosis factor- $\alpha$ and matrix metalloproteinase- 2 levels in an animal model of acne vulgaris. $^{7.8}$ In this study, the two to four sessions of $420-n m$ BBL treatment were performed at a fluence of $5 \mathrm{~J} / \mathrm{cm}^{2}$, a pulse duration of $150 \mathrm{msec}$, and a cooling crystal temperature of $15^{\circ} \mathrm{C}$, and elicited near complete improvement in active acne lesions. We suggest that irradiated blue light provided therapeutic effects by eliminating $P$. acnes and associated inflammatory reactions.

In the treatment of inflammatory skin disorders, BBL with the cut-off wavelength of $590 \mathrm{~nm}$ and pulsed dye laser treatment can decrease inflammatory cell infiltration in the dermis by targeting vascular components. ${ }^{9} \mathrm{~A}$ previous study demonstrated that $\mathrm{BBL}$ treatments at a cut-off of $590 \mathrm{~nm}$, a fluence of 12 to $13 \mathrm{~J} / \mathrm{cm}^{2}$, and a pulse duration of $30 \mathrm{msec}$ in patients with atopic dermatitis significantly reduced not only erythema, but also scaling, inflammatory papules, and lichenification. ${ }^{9}$ In this report, a single pass of $590-\mathrm{nm}$ BBL treatment in step 2 was delivered at a fluence of $15 \mathrm{~J} / \mathrm{cm}^{2}$, a pulse duration of 200 msec, and a cooling crystal temperature of $15^{\circ} \mathrm{C}$ in modification of the second step previously suggested when the 3-step protocol was introduced, which utilized a cut-off filter of 560 or $590 \mathrm{~nm}$, a fluence of $15 \mathrm{~J} / \mathrm{cm}^{2}$, a pulse duration of $15 \mathrm{msec}$, and 2-3 passes. 'By doing so, we noted that our patients were able to better tolerate pain during the procedure, and active acne vulgaris and post-acne erythema were treated effectively.

$\mathrm{BBL}$ treatment has been found to decrease elastotic fibers, increase epidermal thickness, and rearrange collagen fibers in aged cells and tissues. ${ }^{10}$ Furthermore, BBL-induced functional rejuvenation can be achieved by the restoration of gene expression pattern and the structural and functional integrity thereof. ${ }^{10}$ Additionally, the constant motion delivery of BBL energy has been proposed for more balanced, more effective, and safer delivery of BBL energy to target tissue, compared to the static operation technique.' In this study, multiple passes using a constant motion technique with a 590ST cut-off filter, an intensity of $10 \mathrm{~W} / \mathrm{cm}^{2}$, a pulse duration of $12 \mathrm{sec}$, and a cooling crystal temperature of $30^{\circ} \mathrm{C}$ were delivered in step 3 to the patients. We deemed that BBL-induced fibroblast activation and neocollagenesis improved overall skin tone and texture, post-acne erythema, and atrophic scars in our patients, along with clinical improvement of active acne lesions, by delivering polychromatic light sources using the constant motion technique.

In conclusion, we were able to effectively and safely treat two Japanese patients by delivering BBL energy using a modified 3-step protocol. Our patients experienced noticeable clinical improvements in active acne vulgaris, post-acne erythema, and skin tone and texture. We suggest that the irradiation of qualified high-intensity BBL sources using the three different treatment settings can synergistically and simultaneously target the major pathogenic factors of acne vulgaris, and result in satisfactory clinical improvement in inflammatory acne vulgaris and post-acne erythema.

\section{ACKNOWLEDGEMENTS}

We would like to thank Maki Sakamoto (Clinical \& Marketing Manager; Sciton, Tokyo, Japan) and Duke Song (Consultant; Sciton, Palo Alto, CA, USA) for their assistance with technical support. We would also like to thank Anthony Thomas Milliken, ELS (Editing Synthase, Seoul, Koreal for his help with the editing of this manuscript.

\section{REFERENCES}

1. Bitter P Jr. Acne treatment with 3-step broadband light protocol. J Drugs Dermatol 2016;15:1382-8.

2. Seaton ED, Charakida A, Mouser PE, Grace I, Clement RM, Chu AC. Pulsed-dye laser treatment for inflammatory acne vulgaris: randomised controlled trial. Lancet 2003;362:1347-52.

3. Wiznia LE, Stevenson ML, Nagler AR. Laser treatments of active acne. Lasers Med Sci 2017;32:1647-58.

4. Tong LX, Brauer JA. Lasers, Light, and the treatment of acne: a comprehensive review of the literature. J Drugs Dermatol 2017;16:1095-102.

5. Zaenglein AL, Pathy AL, Schlosser BJ, Alikhan A, Baldwin HE, Berson DS, et al. Guidelines of care for the management of acne vulgaris. J Am Acad Dermatol 2016;74:945-73.e33.

6. Ashkenazi H, Malik Z, Harth Y, Nitzan Y. Eradication of Propionibacterium acnes by its endogenic porphyrins after illumination with high intensity blue light. FEMS Immunol Med Microbiol 2003;35:17-24.

7. Omi T, Bjerring P, Sato S, Kawana S, Hankins RW, Honda M. $420 \mathrm{~nm}$ intense continuous light therapy for acne. J Cosmet Laser Ther 2004;6:156-62. 
8. Fan X, Xing YZ, Liu LH, Liu C, Wang DD, Yang RY, et al. Effects of $420-n m$ intense pulsed light in an acne animal model. J Eur Acad Dermatol Venereol 2013;27:1168-71.

9. Oh SH, Bae BK, Kim TG, Kwon YS, Lee JH, Lee KH. Effective treatment of facial redness caused by atopic dermatitis using intense pulsed light systems. Dermatol Surg 2010;36:475-82.

10. Chang AL, Bitter PH Jr, Qu K, Lin M, Rapicavoli NA, Chang HY. Rejuvenation of gene expression pattern of aged human skin by broadband light treatment: a pilot study. J Invest Dermatol 2013;133:394-402. 